ORIGINAL ARTICLE

\title{
Terminology used by pathologists in reporting on sudden infant deaths
}

\section{S R Limerick, C J Bacon}

J Clin Pathol 2004;57:309-311. doi: 10.1136/jcp.2003.013052

See end of article for authors' affiliations .....................

Correspondence to: Dr C J Bacon, Foundation for the Study of Infant Deaths, Artillery House, 111-9 Artillery Row, London SWIP IRT, UK; Chrisandheatherbacon@ ukonline.co.uk

Accepted for publication 2 October 2003

\begin{abstract}
Aim: To determine whether the terms used by pathologists in reporting on sudden infant deaths are applied consistently.

Method: Postal survey.

Results: Replies were received from 63 pathologists who carry out necropsies after sudden infant deaths. There was a pronounced variation in their use of the terms "sudden infant death syndrome" and "unascertained", with the last term being applied not only when there were apparently suspicious features but also in various other circumstances. Opinions were divided as to whether the term "sudden infant death syndrome" still serves a useful purpose. Deaths that had occurred while bed sharing were categorised in several different ways. Many pathologists commented on the inadequacy of the history available to them at the time of their report.

Conclusions: There is a serious need for greater consistency in the way pathologists report on sudden death in infants. Pathologists should be provided with a full history before they carry out the necropsy.
\end{abstract}

$\mathrm{T}$ he term sudden infant death syndrome (SIDS) was introduced in 1969 by Beckwith ${ }^{1}$ to provide a nonpejorative designation for sudden unexpected deaths in infancy (SUDI) that were thought to be natural but could not be explained either by history or by a thorough postmortem examination. In 1971, SUDI (or equivalent) was officially recognised as a registrable cause of death in England and Wales, and once the new term had become established most unexplained sudden infant deaths came to be registered this way. The ninth edition of the International classification of disease (ICD 9), issued in 1979, included a specific code for SIDS (798.0).

Over the past few years, however, there has been debate as to whether the term SIDS still serves a useful purpose, ${ }^{2-4}$ and there is an increasing tendency for some pathologists, when reporting on SUDI for which they cannot identify a specific cause, to use the term "unascertained"; the number of infant deaths so registered has risen from 32 in 1997 to 97 in 2002. Some authorities, for example Green, ${ }^{3}$ have encouraged this tendency on the grounds that a proportion of unexplained SUDI are suspicious, and it has been argued that the characteristics of deaths categorised as unascertained differ from those ascribed to SIDS. ${ }^{5}$ Because there are no agreed criteria for designation as unascertained, there is a need to know whether the term is being applied in a precise and consistent manner.

\section{"Over the past few years, however, there has been debate as to whether the term sudden infant death syndrome still serves a useful purpose"}

Therefore, we undertook a survey among pathologists who carry out SUDI necropsies to determine the circumstances in which they use the term unascertained. At the same time, we took the opportunity to ask about other aspects of their practice with respect to SUDI. The survey was conducted in autumn 2001 by SRL for the Foundation for the Study of Infant Deaths in collaboration with the Office of National Statistics (ONS) and the Royal College of Pathologists.

\section{METHOD}

The survey was carried out by means of a questionnaire sent by post to all the consultant pathologists whom the Royal College of Pathologists had on record as performing SUDI necropsies, whether on a regular or on an occasional basis.

\section{RESULTS}

Questionnaires were sent to 105 pathologists, 68 (65\%) of whom replied. The answers of five were excluded because they said they no longer did SUDI necropsies. Of the remaining 63, 29 were forensic pathologists, 24 paediatric pathologists, and 10 were general histopathologists. Six in the last group also had forensic accreditation and are included with forensic pathologists in the analysis. Table 1 gives the frequency of performance of SUDI necropsies by respondents in each category, expressed as the average annual number over the past three years.

\section{Use of term unascertained}

When asked "If the necropsy fails to reveal an adequate cause of death, might you use the term unascertained?", 11 pathologists said they always did, 19 said frequently (that is, more than half of the time), 26 said occasionally, and seven said never. One in the last group preferred the term "unexplained". Pathologists from all three categories used the term unascertained, forensic pathologists rather more frequently than the others. Table 2 shows the reasons given by the 56 pathologists who always or sometimes used the term; several gave more than one reason.

The most frequent reason for using the term unascertained, cited by over two thirds of the pathologists concerned, was that death had occurred while the baby was sharing a bed with an adult. This reflects current uncertainties about the mode of death in these circumstances. A similar proportion used the term when suspicious features were present. Respondents specified a wide range of features that

Abbreviations: ONS, Office of National Statistics; SIDS, sudden infant death syndrome; SUDI, sudden unexpected deaths in infancy 
Table 1 Frequency of performance of sudden unexplained death in infancy (SUDI) necropsies

\begin{tabular}{llllll}
\hline \multirow{2}{*}{$\begin{array}{l}\text { Type of } \\
\text { pathologist }\end{array}$} & \multicolumn{4}{l}{ Number of SUDI necropsies/year (\% of pathologists) } \\
\cline { 2 - 6 } & $<\mathbf{5}$ & $\mathbf{6 - 1 0}$ & $\mathbf{1 1 - 2 0}$ & $\mathbf{2 0}$ & Total \\
\hline Paediatric & $3(12.5)$ & $\mathbf{7 ( 2 9 . 2 )}$ & $9(37.5)$ & $5(18.3)$ & 24 \\
Forensic & $13(37.1)$ & $12(34.3)$ & $\mathbf{8}(22.9)$ & $2(5.7)$ & 35 \\
General & $2(50)$ & & $2(50)$ & & 4 \\
Total & $18(28.6)$ & $19(30.1)$ & $19(30.1)$ & $\mathbf{7}(11.1)$ & 63 \\
\hline
\end{tabular}

they might regard as suspicious. These included in the history: an unusual or inconsistent account, abuse of drugs or alcohol by a parent, a history of child abuse or previous unexplained infant death in the family, the age of the baby or the time of death not typical for SIDS, previous unexplained illness or apparent life threatening event, and repeated medical attendance for no obvious reason; and at necropsy: bruising or other unexplained marks, bleeding from the mouth and nose, fractures of any age, internal signs of asphyxia, unexplained pulmonary haemorrhage or siderophages. A few admitted that they used the term unascertained to trigger an inquest. Several commented that they used the term unascertained as an honest description when no specific cause of death could be found

\section{Information available to pathologists}

More than half the respondents (32 of the 62 who replied to this question) said that they usually did not have enough information at the time of the necropsy to reach a reliable conclusion about the cause of death. All of these 32 mentioned inadequacies in the history available to themfor example, in the previous medical history (including obstetric and primary care records) and social history, or in the account of the antecedents and circumstances of the death. Fifty two always received a report from the coroner's officer, but only 10 regularly had a report from a health professional. Thirty two pathologists, most of them forensic, stated that they sometimes visited the scene of the baby's death, but none did so in every case. Eleven pathologists also pointed out that full results of ancillary tests, such as some histological and biochemical studies, were not available at the time they were required to make their initial report. Several respondents suggested that a multidisciplinary discussion at which all the relevant professionals could share information was the best way of elucidating the factors contributing to the death.

\section{Use of term sudden infant death syndrome}

When asked whether they thought that the term SIDS still served a useful purpose, 38 pathologists said yes and 24 said no, whereas one was equivocal. Criticisms of the term SIDS included the following: SIDS implies a single disease process and prevents further investigation and enquiries; SIDS is a diagnosis of exclusion and therefore a lazy way of saying "I don't know what happened"; the term served a useful purpose but I wish it had never been introduced, because the search for natural disease has been wasteful and death scene investigation, where the key to most cases lies, has been prevented.

A quarter of the respondents said that if a specific condition, such as bronchitis, was evident at necropsy but did not constitute an adequate cause of death, they would sometimes put that condition in their report in addition to the term SIDS. When asked what term they would use if a baby died unexpectedly while sharing a bed with an adult and no specific cause was found at necropsy, 38 pathologists
Table 2 Reasons cited for categorising a death as unascertained

\begin{tabular}{lll}
\hline Reason & $\begin{array}{l}\text { Number of } \\
\text { citings }\end{array}$ & $\begin{array}{l}\text { Percent of } \\
\text { pathologists }\end{array}$ \\
\hline Coroner's preference & 2 & $4 \%$ \\
Pathologist's preference & 30 & $54 \%$ \\
Presence of suspicious features & 37 & $66 \%$ \\
Presence of avoidable risk factors & 10 & $18 \%$ \\
To encourage inquest & 8 & $14 \%$ \\
$\begin{array}{l}\text { To allow retention of tissue for } \\
\text { histology }\end{array}$ & 2 & $4 \%$ \\
Death occurred while sharing bed \\
with adult
\end{tabular}

said unascertained, 25 said SIDS, six said SUDI (or equivalent), whereas one said asphyxia, several commenting that it would depend on the precise circumstances (hence the total of more than 63).

\section{DISCUSSION}

Our response rate of $65 \%$ was disappointing but statistically acceptable, and we have no reason to think that the sample was not representative of pathologists who currently carry out SUDI necropsies. It is interesting that the proportion of general pathologists was considerably lower than that of paediatric and forensic pathologists, contrasting with the findings of Woolf ${ }^{6}$ and the CESDI SUDI studies, ${ }^{7}$ in which SUDI necropsies were apportioned about equally between the three types of pathologist. This finding could result from a lower response to the survey by general pathologists. However, if response rates were equivalent, it would suggest that, following the fall in the incidence of SUDI, coroners now instruct specialist pathologists, whether forensic or paediatric, in a higher proportion of cases than in the past. Nevertheless, over a quarter of all respondents were performing fewer than six SUDI necropsies each year. Infrequent practice by individual pathologists hinders the development of expertise in this extremely difficult field, and greater concentration with specialists would probably bring better quality and consistency.

Despite the conclusion reached by the CESDI SUDI studies that the most useful component of the investigation of SUDI is a detailed clinical history, ${ }^{7}$ half the respondents in our survey said that the history available to them was inadequate. This again emphasises the need for a paediatrician (or other suitably qualified health professional) to be involved in the investigation of SUDI from the outset, so that a detailed history can be compiled from interview with the family, consultation with relevant professionals, inspection of the death scene, and review of the medical records. This must be done very quickly so that at least an interim report is available for the pathologist at the time of the necropsy. This task requires paediatric training and experience, and it is unreasonable to delegate it to a coroner's officer. It was interesting that several pathologists, most of them forensic, said that they sometimes visited the death scene. This would complement a paediatric report and would be particularly helpful if none was available.

\section{"Designation as unascertained may attach a stigma to the death, which can be hurtful and damaging for the family"}

The survey showed wide variations between pathologists in the terms they use when reporting on SUDI for which they could not find a specific cause, in particular in their use of the term unascertained. This variation in terminology appears to 
reflect varying attitudes to the concept of SIDS. Some dislike this designation on the grounds that it implies a specific diagnosis where none can be found, and think it is more honest always to call such a death unascertained. Most use the term unascertained selectively, but the variety of criteria given in table 2 shows that deaths so categorised are heterogeneous and by no means always suspicious. Some pathologists do not use the term unascertained at all. The definition of SIDS requires that no specific cause can be found, so in this respect there is no difference between SIDS and unascertained. However an essential criterion for designation as SIDS is that the death should be deemed natural, whereas unascertained leaves open the possibility of it being unnatural. Thus, designation as unascertained may attach a stigma to the death, which can be hurtful and damaging for the family. It was mainly to avoid such stigma that the concept of SIDS was originally introduced.

It is generally accepted that a small proportion of SUDI do indeed arise from maltreatment by parents, but their identification is often very difficult and can only be made after a most thorough investigation. ${ }^{4}$ It is prejudicial to attempt to make a distinction between natural and suspicious on the basis of the incomplete information available immediately after the necropsy. Premature categorisation is likely to result in mistakes-in either direction. It would be more equitable if all unexplained SUDI were given the same initial designation, and the distinction made only after the entire investigation, which in many cases will include an inquest, has been completed. The circumstances in which the designation SIDS should be used is still the subject of active debate. $^{8}$

Each year, the ONS records about 30 infant deaths for which the pathologist has given a specific condition in addition to a term such as sudden infant death. This survey shows that the pathologists who do this usually consider the specific condition to be coincidental or contributory rather than causative, so that the death should properly be categorised as SIDS. However, under the international rules for coding, ONS is obliged to allocate such cases to the more specific condition and cannot include them in the code for SIDS (ICD 9, 798.0). Therefore, a pathologist who thinks the death was SIDS but also includes a specific condition in his summary is, so far as ICD coding is concerned, nullifying his diagnosis. Partly for this reason, since 1971, ONS has published totals for a broader category of "sudden infant deaths", which includes all cases where there is any mention of sudden infant death (or equivalent) on the certificate.

Whatever is decided about the terms that should be used, it is essential that they should be applied in a more consistent

\section{Take home messages}

- Pathologists varied greatly in their use of the terms "sudden infant death syndrome" and "unascertained", with the last term being applied not only when there were suspicious features but also in a variety of other circumstances

- Opinions were divided as to whether the term "sudden infant death syndrome" still serves a useful purpose

- Greater consistency is needed in the way pathologists report on sudden death in infants

- Pathologists should be provided with a full history before they carry out the necropsy

manner. The variations shown in this survey arise from poor consensus among practitioners, lack of central guidance, and absence of audit. Without these constraints subjectivity has free rein, so that at present death certification for SUDI depends partly on postal code. This inconsistency is confusing and sometimes damaging for families, and undermines accurate statistical assessment. Improvement might best be achieved if these problems could be addressed in the current review of the coroners' system.

\section{Authors' affiliations}

S R Limerick, C J Bacon, Foundation for the Study of Infant Deaths, Artillery House, 111-9 Artillery Row, London SW IP 1RT, UK

Lady Limerick is vice-chair and $\operatorname{Dr}$ Bacon is medical adviser to the Foundation for the Study of Infant Deaths, a charity that promotes research into infant deaths and supports bereaved parents.

\section{REFERENCES}

1 Beckwith JB. Discussion of terminology and definition of the sudden infant death syndrome. In: Bergman JB, Ray CG, eds. Proceedings of the second international conference on causes of sudden death in infants. Washington: University of Washington Press, 1970:14-22.

2 Meadow R. Unnatural sudden infant death. Arch Dis Child 1999;80:7-14.

3 Green MA. Time to put "cot death" to bed. BMJ 1999:319:697-8.

4 Limerick S. Not time to put "cot death" to bed. BMJ 1999;319:698-700.

5 Office for National Statistics. Are unascertained deaths the same as sudden infant deaths? Health Statistics Quarterly 2001;10:20-4.

6 Woolf M. Coroners' post mortems in England and Wales; a report for the Department of Health. London: Office for National Statistics, Social Survey Division, 1997

7 Fleming $\mathrm{P}$, Bacon $\mathrm{C}$, Blair $\mathrm{P}$, et al. Sudden unexpected deaths in infancy: the CESDI SUDI studies 19939-6. London: Stationery Office, 2000

8 Beckwith JB. Defining the sudden infant death syndrome. Arch Pediatr Adolesc Med 2003; 157:286-90. 